\title{
KINETIC STUDY ON EXTENDED RELEASE OF THEOPHYLLINE CAPLET WITH DIFFERENT BRANDS HYPROMELLOSE MATRIX
}

\author{
Marline Abdassah, Yoga Windhu Wardhana*, Taofik Rusdiana, Maria Z.A. Yordan, \\ Feni D. Anggraini and Moeljadi G. Tedjasaputra \\ Department of Pharmaceutics and Pharmaceutical Technology, Faculty of Pharmacy, Universitas \\ Padjadjaran, Jatinangor-45363, Indonesia \\ *E-mail: ayodhna@gmail.com \\ Tel.: +62227796200 .
}

Received: Jan 01, 2016 / Revised: Jan 14, 2016 / Accepted: Jan 16, 2016

The aim of the present investigation was to prepare and evaluate kinetics of extended release formulations of theophylline using Methocel K4M CR Premium/MK and Metolose 90SH-4000SR/M9. All formulated tablets with various concentrations $(\% 4.0 ; 4.25 ; 4.5 ; 4.75 ; 5.0)$ of hypermellose were prepared by wet granulation method and found to be complied with the official requirements. The results showed the zero order kinetics and similarity (f2>50) with the marketed brand product were indicated by the formulations containing MK 4.75\% - MK 5.0\% and M9 5\%. It was concluded that the higher the hypromellose concentration the slower the release of drug.

Key words: Theophylline, Hypromellose, Extended release kinetics, Independent parameters.

\section{INTRODUCTION}

For very small dosage administration of drugs resulting in restrictive absorption, the usage of controlled release is better suited (Bhardwaj et al 2000). Theophylline, which has short half-life, belongs to narrow therapeutic index Drug (Bayomi et al 2001). To this character, extended release formulation should be prepared to achieve adequate blood levels and maintained with a minimal fluctuations. Meanwhile, administration of such dosage form often reduce patient disobedience, resulting in attaining effective therapy.

Although many researchers reported multiparticulate and other sustained release formulations (Dahiya et al 2008; Dahiya and Tyagi, 2008; Dahiya and Gupta, 2011; Basarkar et al 2013; Tyagi and Kori, 2013; Verma et al 2014; Nagpal et al 2014; Dahiya and Onker, 2015), matrix tablet has also been found as one of the most significant extended release pharmaceutical formulation. Extended release formulations generally formulated as coated tablet, consist of complex and expensive steps. Hydroxypropyl Methyl Celullose (HPMC) or Hypromellose with special substitution site have been commonly used in simple controlled release formulations (Ishikawa et al 2000; Saiful et al 2010; Sultana and Khosru, 2012; Abdassah et al 2015). This is based on highly adsorbable hydrogelling capacity in the matrix as viscosity barrier for drug release (Shin-Etsu, 2005). In this study, we used hypromellose from different origin and having similar grade : Methocel K4MCR Premium (Dow), Metolose 90SH-4000SR (Shin-Etsu) for comparing drug release kinetic profiles and theophylline retarded caplet (market derived) as standard quality evaluation. This paper depicts the kinetic release profile of theophylline extended release formula from different origin hypromellose matrices, labscale manufacturing and testing the release behavior of these products using dependent methods (mathematical model) and independent 
methods [similarity factor (f2), difference factor (f1)]. The purpose of this work was to produce theophylline extended release caplets using difference hypromellose matrix and to evaluate the release kinetic profile.

\section{MATERIALS AND METHODS}

The following materials were obtained from commercial sources: Theophylline anhydrous (ex Jilin Shulan, China), Metolose 90SH-4000SR (ex ShinEtsu, Japan), Methocel K4M-CR Premium (ex Dow Chemical, USA), Lactose-SD (ex Grande, USA), Talcum (ex Haicen, China) and Magnesium stearate (ex Faci, Indonesia), marketed theophylline retard caplets, artificial gastric fluid $\mathrm{pH} 1.2$ and artificial intestinal fluid $\mathrm{pH} 6.0$ without enzymes (attempt to match USP XXX NF $\mathrm{XXV}$ ) and reagents (Merck, appropriate analysis grade).

\section{Preparation of theophylline extended release caplet}

All caplets were prepared by wet granulation method. The compositions of Theophylline ER matrix caplets are given in the Table 1 . Theophylline and Lactose SD were mixed together. Hypromellose was dissolved in a portion of water to make a $10 \%$ binder solution. Which then added to the mixed powder, mixed well to form a coherent mass. This mass was then passed through no. 10 sieving mesh and resultant granules were dried in the oven at $50 \pm 5^{\circ} \mathrm{C}$ for $24 \mathrm{~h}$. The dried granules were further passed through no. 16 sieving mesh.

The dried granules were then characterized, mixed with lubricant (magnesium stearate and talcum) and then som-pressed to form $400 \mathrm{mg}$ caplets using single punch tablet machine (Korsch, Tipe EK 0).

Table 1. Composition of Theophylline ER matrix caplets (mg/tablet)

\begin{tabular}{|c|c|c|c|c|c|}
\hline \multirow{2}{*}{ Composition } & \multicolumn{5}{|c|}{ Formulation } \\
\cline { 2 - 6 } & $\mathbf{F 1 ~ ( 4 \% )}$ & $\mathbf{F 2 ~ ( 4 . 2 5 \% )}$ & F3 (4.5\%) & F4 (4.75\%) & F5 (5\%) \\
\hline Theophylline (mg) & 300 & 300 & 300 & 300 & 300 \\
\hline Lactose SD (mg) & 78 & 77 & 76 & 75 & 74 \\
\hline Hypromellose* (mg) & 16 & 17 & 18 & 19 & 20 \\
\hline Mg. Stearate (mg) & 4 & 4 & 4 & 4 & 4 \\
\hline Talcum (mg) & 2 & 2 & 2 & 2 & 2 \\
\hline Total weight (mg) & 400 & 400 & 400 & 400 & 400 \\
\hline
\end{tabular}

*Hypromellose : Metolose 90SH-4000SR (M9) and Methocel K4M-CR Premium (MK)

\section{Evaluation of granules}

The granules were evaluated for loss on drying, flowability, compressability (Carr's index), and angle of repose.

\section{Evaluation of caplets}

The prepared caplets were evaluated for content uniformity, weight uniformity, size uniformity, hardness, friability, and in vitro drug release.

\section{In vitro drug release study}

In vitro drug release profile was carried out as per USP XXX NF XXV using Sotax AG CH-4008 BASEL, Tipe AT- 6 apparatus (paddle type). The dissolution studies were performed using 900 $\mathrm{ml}$ of artificial gastric fluid $\mathrm{pH} 1.2$ at $37 \pm 0.5^{\circ} \mathrm{C}$ and $50 \mathrm{rpm}$. Each $10 \mathrm{ml}$ sample was drawn at 5 , $10,15,30,45,60 \mathrm{~min}$, replaced with the artificial intestinal fluid pH 6.0 without enzymes. Ten ml samples at $90,120,150,180,240,300$, $360,420,480$ minute were withdrawn and replaced with same quantity of fresh dissolution medium each time. Collected samples were suitably diluted with dissolution medium and analyzed at $269 \mathrm{~nm}$, using fresh dissolution medium as blank in UV-double beam spectrophotometer.

\section{Drug release kinetic profile}

One way ANOVA tool was used to analyze the concentration variation of hypromellose to caplets release data. If there are any effect which influenced the dissolution mechanism, it would be studied by plotting dissolution profiles in various kinetic models i.e. zero order, first order and higuchi model. (dependent methods). To know which of the formulation possessed the most similar release behaviour to the marketed products, the difference factors $\left(f_{1}\right)$ and similarity factors $\left(f_{2}\right)$ are recommended which are computed by independent methods.

\section{RESULTS AND DISCUSSION Granules properties}

The granules of all formulations exhibited good quality as summarized in Table 2a,b. All granules were found suitable to the compression requirement (Aulton, 2002). 
Table 2a. Properties of theophylline matrix granules (F1-F3)

\begin{tabular}{|c|c|c|c|c|c|c|c|}
\hline \multirow{2}{*}{ Parameters } & \multicolumn{2}{|c|}{ F1 } & \multicolumn{2}{c|}{ F2 } & \multicolumn{2}{c|}{ F3 } & Req. \\
\cline { 2 - 8 } & M9 & MK & M9 & MK & M9 & MK & 1.02 \\
\hline $\begin{array}{c}\text { Loss on } \\
\text { drying (\%) }\end{array}$ & 0.55 & 0.50 & 0.77 & 0.66 & 0.64 & $<2$ \\
\hline $\begin{array}{c}\text { Angle of } \\
\text { repose ( ) }\end{array}$ & $19.13 \pm 0.95$ & $14.21 \pm 0.54$ & $19.98 \pm 0.41$ & $14.17 \pm 0.48$ & $19.43 \pm 0.63$ & $12.66 \pm 1.45$ & $<25$ \\
\hline $\begin{array}{c}\text { Flowability } \\
\text { g/detik) }\end{array}$ & $14.78 \pm 0.42$ & $18,98 \pm 0.66$ & $14.02 \pm 0.28$ & $19.86 \pm 0.53$ & $14.67 \pm 1.01$ & $20.06 \pm 0.96$ & $>10$ \\
\hline $\begin{array}{c}\text { Carr index } \\
(\%)\end{array}$ & $13.82 \pm 2.01$ & $12.67 \pm 0.61$ & $14.67 \pm 2.18$ & $14.64 \pm 2.74$ & $13.44 \pm 1.36$ & $14.13 \pm 2.55$ & $5-18$ \\
\hline
\end{tabular}

Table 2b. Properties of theophylline matrix granules (F4-F5)

\begin{tabular}{|c|c|c|c|c|c|}
\hline \multirow{2}{*}{ Parameters } & \multicolumn{2}{|c|}{ F4 } & \multicolumn{2}{c|}{ F5 } & \multirow{2}{*}{ Req. } \\
\cline { 2 - 5 } & M9 & MK & M9 & MK & $<2$ \\
\hline $\begin{array}{c}\text { Loss on } \\
\text { drying (\%) }\end{array}$ & 0.47 & 0.84 & 0.37 & 0.60 & $<25$ \\
\hline $\begin{array}{c}\text { Angle of } \\
\text { repose ( })\end{array}$ & $18.97 \pm 0.75$ & $14.90 \pm 0.46$ & $18.16 \pm 0.40$ & $13.51 \pm 0.09$ & $>10$ \\
\hline $\begin{array}{c}\text { Flowability } \\
\text { (g/detik) }\end{array}$ & $15.04 \pm 0.80$ & $19.68 \pm 0.64$ & $15.92 \pm 0.3$ & $18.31 \pm 1.50$ & $5-18$ \\
\hline $\begin{array}{c}\text { Carr index } \\
(\%)\end{array}$ & $12.15 \pm 1.15$ & $16.92 \pm 2.21$ & $9.90 \pm 1.35$ & $12.29 \pm 1.80$ & \\
\hline
\end{tabular}

\section{Caplets properties}

Results of evaluation suggested that all caplet formulations were complied to the official friability specifications, as summarized in the Table 3a,b. This means all formulations did not affect theophylline release in the caplets form.
The contents uniformity of the drug in the caplets was complied to the USP XXX and NF XXV.

The content uniformity for the marketed product has also been fulfilled to the specification $(100.63 \pm 1.70 \%)$.

Table 3a. Properties of theophylline caplets (F1-F3)

\begin{tabular}{|c|c|c|c|c|c|c|c|}
\hline \multirow{2}{*}{ Parameters } & \multicolumn{2}{|c|}{ F1 } & \multicolumn{2}{|c|}{ F2 } & \multicolumn{2}{|c|}{ F3 } & \multirow{2}{*}{ Req. } \\
\hline & M9 & MK & M9 & MK & M9 & MK & \\
\hline $\begin{array}{l}\text { Length } \\
(\mathrm{mm})\end{array}$ & $16.94 \pm 0.01$ & $16.7 \pm 0.008$ & $16.92 \pm 0.01$ & $16.96 \pm 0.014$ & $16.99 \pm 0.01$ & $16.97 \pm 0.007$ & - \\
\hline $\begin{array}{l}\text { Width } \\
\text { (mm) }\end{array}$ & $6.46 \pm 0.01$ & $6.50 \pm 0.005$ & $6.44 \pm 0.01$ & $6.50 \pm 0.016$ & $6.51 \pm 0.003$ & $6.50 \pm 0.007$ & - \\
\hline $\begin{array}{c}\text { Thickness } \\
(\mathrm{mm})\end{array}$ & $3.70 \pm 0.02$ & $3.78 \pm 0.15$ & $3.70 \pm 0.02$ & $3.78 \pm 0.070$ & $3.75 \pm 0.02$ & $3.93 \pm 0.045$ & - \\
\hline $\begin{array}{l}\text { Weight } \\
\text { (mg) }\end{array}$ & $407.03 \pm 2.06$ & $406 \pm 2.58$ & $408.34 \pm 5.61$ & $409.41 \pm 4.19$ & $406.74 \pm 4.42$ & $414.4 \pm 4.68$ & - \\
\hline $\begin{array}{l}\text { Hardness } \\
(\mathrm{N})\end{array}$ & $125.88 \pm 13.21$ & $114.4 \pm 17.4$ & $128.75 \pm 7.84$ & $130.55 \pm 20.8$ & $130.75 \pm 10.79$ & $131 \pm 18.6$ & - \\
\hline $\begin{array}{c}\text { Friability } \\
(\%)\end{array}$ & $0.401 \pm 0.003$ & $0.52 \pm 0.06$ & $0.396 \pm 0.050$ & $0.37 \pm 0.05$ & $0.374 \pm 0.030$ & $0.50 \pm 0.15$ & $<1 \%$ \\
\hline $\begin{array}{c}\text { Contents } \\
(\%)\end{array}$ & $99.67 \pm 1.45$ & $103.13 \pm 3.15$ & $98.66 \pm 1.23$ & $101.35 \pm 2.3378$ & $100.75 \pm 0.55$ & $96.96 \pm 0.4972$ & $90-110$ \\
\hline
\end{tabular}

\section{In vitro drug release profiles}

Release patterns of different hypromellose origin and the marketed theophylline retard (as working standard), was conformed to the USP XXX NF XXV. The results suggested that all formulations met official specifications (Table 4a,b). One way ANOVA also showed that difference in hypromellose concentrations provided different drug release profiles. With higher hypromellose concentration, more extended drug release was achieved. The release patterns of each formulations of the same origin (Figure 1A, 1B), looked alike but when analyzed with the release kinetics and independent parameters (Table 5, 6), they were found to exhibit different kinetic order and independent parameters compared to that of the marketed theophylline preparation. 
Table 3b. Properties of theophylline caplets (F4-F5)

\begin{tabular}{|c|c|c|c|c|c|}
\hline \multirow{2}{*}{ Parameters } & \multicolumn{2}{|c|}{ F4 } & \multicolumn{2}{|c|}{ F5 } & \multirow{2}{*}{ Req. } \\
\hline & M9 & MK & M9 & MK & \\
\hline $\begin{array}{l}\text { Length } \\
\text { (mm) }\end{array}$ & $16.99 \pm 0.01$ & $16.97 \pm 0.006$ & $17.00 \pm 0.01$ & $16.97 \pm 0.023$ & - \\
\hline $\begin{array}{l}\text { Width } \\
\text { (mm) }\end{array}$ & $6.51 \pm 0.01$ & $6.50 \pm 0.005$ & $6.52 \pm 0.01$ & $6.49 \pm 0.030$ & - \\
\hline $\begin{array}{l}\text { Thickness } \\
\text { (mm) }\end{array}$ & $3.71 \pm 0.04$ & $3.86 \pm 0.059$ & $3.92 \pm 0.05$ & $3.74 \pm 0.088$ & - \\
\hline $\begin{array}{l}\text { Weight } \\
\text { (mg) }\end{array}$ & $402.54 \pm 5.05$ & $408.61 \pm 4.32$ & $403.2 \pm 4.41$ & $405.16 \pm 2.79$ & - \\
\hline $\begin{array}{l}\text { Hardness } \\
(\mathrm{N})\end{array}$ & $143.25 \pm 10.95$ & $126.58 \pm 22.4$ & $143.75 \pm 8.68$ & $126.00 \pm 20.1$ & - \\
\hline $\begin{array}{c}\text { Friability } \\
(\%)\end{array}$ & $0.352 \pm 0.022$ & $0.37 \pm 0.08$ & $0.350 \pm 0.019$ & $0.37 \pm 0.03$ & $<1 \%$ \\
\hline $\begin{array}{c}\text { Contents } \\
(\%)\end{array}$ & $100.10 \pm 1.73$ & $97.65 \pm 2.2746$ & $98.43 \pm 1.30$ & $102.29 \pm 2.2026$ & $90-110$ \\
\hline
\end{tabular}

Table 4a. Percentages of drug release from different hypromellose and marketed product (F1-F3)

\begin{tabular}{|c|c|c|c|c|c|c|c|c|}
\hline \multirow{3}{*}{$\begin{array}{l}\text { Time } \\
(\mathrm{min})\end{array}$} & \multicolumn{7}{|c|}{$\%$ Drug release $\left(\right.$ mean $\left._{ \pm} \mathrm{SD}\right)$} & \multirow{3}{*}{$\begin{array}{c}\text { USP Req. } \\
(\%)\end{array}$} \\
\hline & \multicolumn{2}{|c|}{ F1 } & \multicolumn{2}{|c|}{ F2 } & \multicolumn{2}{|c|}{ F3 } & \multirow{2}{*}{ Theo. Std. } & \\
\hline & M9 & MK & M9 & MK & M9 & MK & & \\
\hline 60 & $34.03 \pm 1.4$ & $31.56 \pm 1.27$ & $31.45 \pm 0.77$ & $27.26 \pm 1.92$ & $25.86 \pm 0.36$ & $25.86 \pm 0.74$ & $22.61 \pm 3.62$ & $3-15$ \\
\hline 120 & $42.36 \pm 2.36$ & $41.72 \pm 3.03$ & $40.57 \pm 2.55$ & $34.90 \pm 1.88$ & $34.46 \pm 5.29$ & $33.44 \pm 3.52$ & $26.95 \pm 5.37$ & $20-40$ \\
\hline 240 & $83.20 \pm 4.27$ & $78.05 \pm 1.87$ & $81.10 \pm 3.37$ & $71.94 \pm 0.35$ & $71.14 \pm 4.68$ & $67.77 \pm 7.45$ & $50.05 \pm 6.7$ & $50-75$ \\
\hline 360 & $97.02 \pm 2.26$ & $87.96 \pm 2.77$ & $93.10 \pm 0,14$ & $88.92 \pm 1.41$ & $82.95 \pm 1.52$ & $84.45 \pm 4.52$ & $70.11 \pm 11.56$ & $65-100$ \\
\hline 480 & $97.65 \pm 0.28$ & $93.37 \pm 2.09$ & $95.40 \pm 1.75$ & $91.66 \pm 2.69$ & $89.89 \pm 0.09$ & $90.43+0.99$ & $85.11 \pm 6.82$ & $>80$ \\
\hline
\end{tabular}

Table 4b. Percentages of drug release from different hypromellose and marketed product (F4-F5)

\begin{tabular}{|c|c|c|c|c|c|c|}
\hline \multirow{2}{*}{ Time (min) } & \multicolumn{5}{|c|}{ \% Drug release (mean \pm SD) } & \multirow{2}{*}{ USP Req. } \\
\cline { 2 - 6 } & \multicolumn{2}{|c|}{ F4 } & MK & M9 & MK & \multirow{2}{*}{ Theo. Std. } \\
\cline { 2 - 6 } & $1 \%$ ) \\
\hline 60 & $19.73 \pm 0.30$ & $20.88 \pm 0.20$ & $14.79 \pm 0.59$ & $14.97 \pm 0.88$ & $22.61 \pm 3.62$ & $3-15$ \\
\hline 120 & $31.25 \pm 2.57$ & $25.62 \pm 0.33$ & $28.71 \pm 4.22$ & $24.29 \pm 1.59$ & $26.95 \pm 5.37$ & $20-40$ \\
\hline 240 & $58.81 \pm 1.07$ & $53.85 \pm 4.69$ & $51.22 \pm 2.97$ & $51.25 \pm 3.98$ & $50.05 \pm 6.7$ & $50-75$ \\
\hline 360 & $77.05 \pm 2.05$ & $71.27 \pm 2.15$ & $69.25 \pm 0.88$ & $71.39 \pm 2.55$ & $70.11 \pm 11.56$ & $65-100$ \\
\hline 480 & $87.21 \pm 1.95$ & $85.57 \pm 2.69$ & $83.46 \pm 1.81$ & $84.68 \pm 1.67$ & $85.11 \pm 6.82$ & $>80$ \\
\hline
\end{tabular}

Table 5. Kinetic parameters of different hypromellose and marketed product

\begin{tabular}{|c|c|c|c|c|c|c|}
\hline \multirow{2}{*}{ Formulation } & \multicolumn{6}{|c|}{ Regression Correlation (r') } \\
\cline { 2 - 7 } & \multicolumn{2}{|c|}{ 0th Order } & \multicolumn{2}{c|}{ 1st Order } & \multicolumn{2}{c|}{ Higuchi } \\
\cline { 2 - 7 } & M9 & MK & M9 & MK & M9 & MK \\
\hline F1 & 0.9151 & 0.9164 & 0.9574 & 0.9833 & 0.9785 & 0.9805 \\
\hline F2 & 0.9224 & 0.9432 & 0.9708 & 0.9734 & 0.9792 & 0.9708 \\
\hline F3 & 0.9419 & 0.9497 & 0.9896 & 0.9855 & 0.9786 & 0.9754 \\
\hline F4 & 0.9730 & 0.9797 & 0.9912 & 0.9688 & 0.9796 & 0.9683 \\
\hline F5 & 0.9894 & 0.9864 & 0.9821 & 0.9745 & 0.9693 & 0.9618 \\
\hline Theo. Std. & 0.9855 & 0.9855 & 0.9711 & 0.9711 & 0.9725 & 0.9725 \\
\hline
\end{tabular}


Table 6. Independent parameters of different formulations and marketed product

\begin{tabular}{|c|c|c|c|c|}
\hline \multirow{2}{*}{ F : Theo. Std } & \multicolumn{2}{|c|}{ Similarity factor $\left(\mathbf{f}_{\mathbf{2}}\right)$} & \multicolumn{2}{c|}{ Difference factor $\left(\mathbf{f}_{\mathbf{1}} \mathbf{)}\right.$} \\
\cline { 2 - 5 } & M9 & MK & M9 & MK \\
\hline F1 & $36.18 \pm 2 . .32$ & $40.60 \pm 2.26$ & $43.07 \pm 2.46$ & $34.86 \pm 2.07$ \\
\hline F2 & $38.55 \pm 1.89$ & $45.37 \pm 1.04$ & $38.44 \pm 2.07$ & $25.90 \pm 0.76$ \\
\hline F3 & $49.29 \pm 3.67$ & $49.83 \pm 5.87$ & $21.48 \pm 2.79$ & $20.16 \pm 4.69$ \\
\hline F4 & $64.94 \pm 1.98$ & $79.62 \pm 2.67$ & $10.38 \pm 1.68$ & $4.61 \pm 2.36$ \\
\hline F5 & $78.53 \pm 2.97$ & $74.86 \pm 2.38$ & $4.48 \pm 2.78$ & $5.56 \pm 2.09$ \\
\hline
\end{tabular}
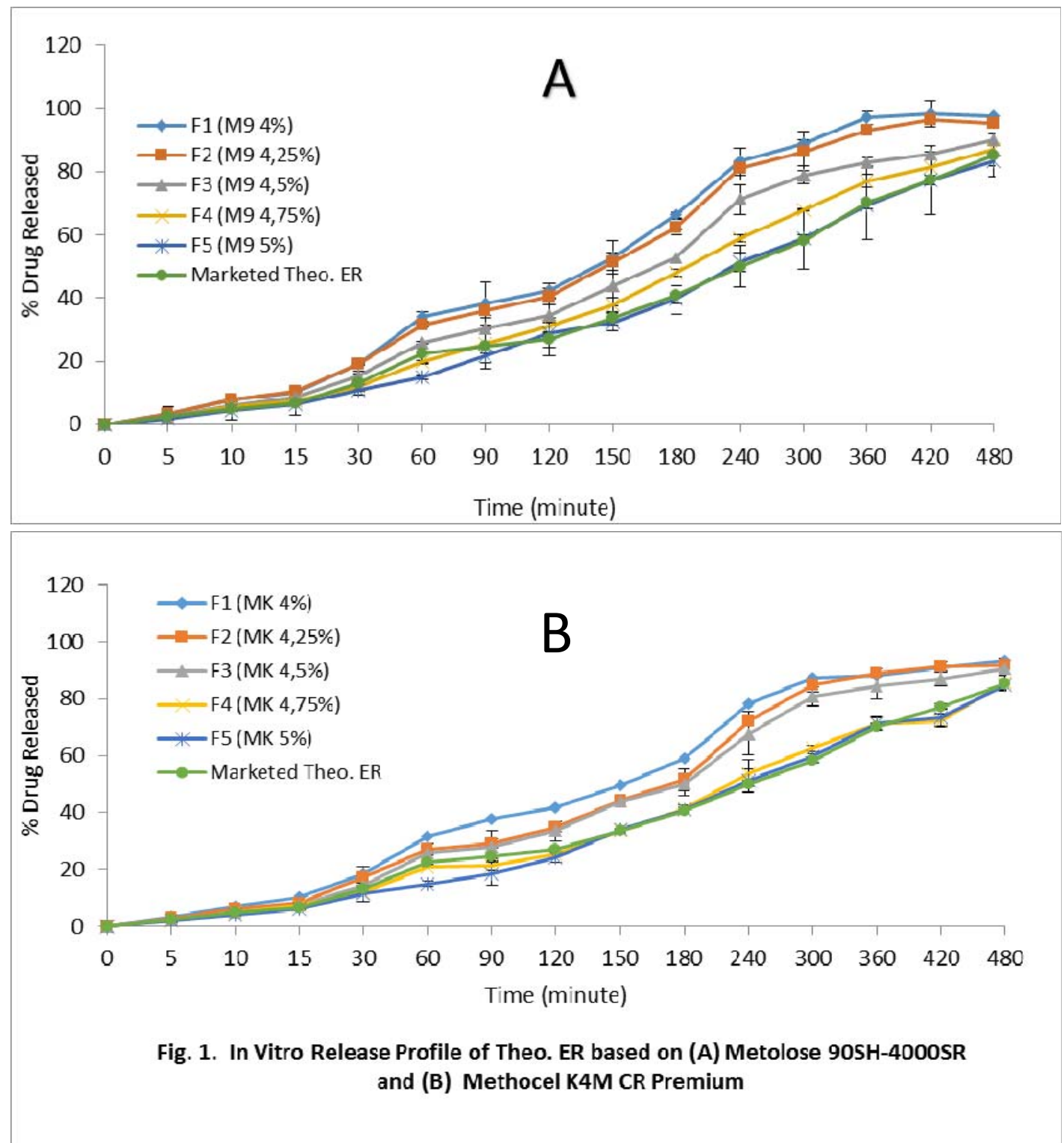

\section{Kinetic release analysis}

Significant difference on theophylline content from both hypromellose origin was not found with One-way ANOVA $(\alpha=0.05)$. However, results of Games-Howell Test showed that there was significant difference between M9 and MK. When all release data were plotted using kinetic equation models (zero order, first order and higuchi model), it was indicated that data obtained from the kinetic release analysis of caplets F4 and F5 were closed to that of marketed theopylline product as indicated by regression coefficient (Table 5). These data have been relatively closed to the resume with independent methods which are compiled in Table 6. There had the similarity and difference factors on its equality which was achieved with F4 and F5.

\section{Conclusion}

Theophylline as extended release formulation with different hypromellose origin, may thus be 
recommended as an oral delivery system to extend drug release for more than $8 \mathrm{~h}$. Although all of the formulation provided extended release of theophylline, caplets prepared with

\section{References}

Abdassah M, Wardhana YW, Priambodo D, Chandra RAI, Herdiana Y, Ch. A. Dissolution profile of theophylline extended release caplets based on metolose $90 \mathrm{SH}-$ 4000SR with accelerated stability study. Actapharmica 2015;2(1):88-92.

Aulton ME. Pharmaceutics: The Science of Dosage Form Design, Churchill Livingstone: New York, 2002; 133-614.

Bhardwaj TR, Kanwar M, Lal R, Gupta A. Natural gums and modified gums as sustained-release carriers. Drug Dev. Ind. Pharm. 2000;26(10):1025-38. [D OI: 10.1081/DDC-10 0100266]

Basarkar GD, Shirsath GN, Patil SB. Development of microspheres containing diclofenac diethylamine as sustained release topical formulation. Bull. Pharm. Res.2013;3(1):14-22.

Bayomi MA, Al-Suwayeh SA, El-Helw A-RM. Excipientexcipient interaction in the design of sustained-released theophylline tablets : in vitro and in vivo evaluation. Drug Dev. Ind. Pharm. 2001;27(6):499-506. [DOI: 10.1081/DD C-100105174]

Dahiya S, Gupta ON. Formulation and in vitro evaluation of metoprolol tartrate microspheres. Bull. Pharm. Res. 2011; 1(1):31-9.

Dahiya S, Onker R. Influence of polymethacrylates and compritol on release profile of a highly water soluble drug metformin hydrochloride. Ars Pharm. 2015;56(1):24-31. [DOI: 10.4321/S2340-98942015000100004]

Dahiya S, Pathak K, Sharma R. Development of extended release coevaporates and coprecipitates of promethazine $\mathrm{HCl}$ with acrylic polymers: formulation considerations. Chem. Pharm. Bull. (Tokyo) 2008;56(4):504-8. [DOI: 10.1248/cpb. hypromellose at $4.75-5 \%$ concentration was found to be the closest to the marketed product with respect to order of drug release and similarity factor.

56.504]

Dahiya S, Tyagi L. Preparation and evaluation of oxytetracycline hydrochloride microbeads for delayed release. Pak. J. Pharm. Sci. 2008;21(2):103-8.

Ishikawa T, Watanabe $\mathrm{Y}$, Takayama K, Endo H, Matsumoto M. Effect of hydroxypropylmethylcellulose (HPMC) on the release profiles and bioavailability of a poorly watersoluble drug from tablets prepared using macrogol and HPMC. Int. J. Pharm. 2000;202(1-2):173-8. [DOI: 10.101 6/S0378-5173(00)00426-9]

Nagpal N, Arora M, Rahar S, Rageeb M, Swami G. Formulation and evaluation of sustained release floating microballoons of ketorolac trometamol. Bull. Pharm. Res. 2014;4(2):86-93.

Saiful MD, Fariba K, Reza UJ. Sustain release theophyllin matrix tablets prepared by using direct compression. Bangladesh Pharm. J. 2010;13(1):42-8.

Sultana S, KH, Khosru. Development and evaluation of invitro release kinetics of sustained release pellets of gliclazide using combinations of cellulose polymers. $J$. Pharm. Educ. Res. 2012;3(1):1-9.

Shin-Etsu Chemical Co., Ltd. Metolose SR. Tokyo: Cellulose \& Pharmaceutical Excipients Department, 2005;1-17.

Tyagi LK, Kori ML. Formulation and in vitro evaluation of Eudragit ${ }^{\circledR}$ RS 100 microspheres containing lornoxicam prepared by emulsion-solvent evaporation method. Bull. Pharm. Res. 2013;3(3):112-20.

Verma S, Kumar V, Jyoti, Mishra DN. Formulation, evaluation and optimization of mucoadhesive microspheres of acyclovir. Bull. Pharm. Res. 2014;4(1): 14-20. 